\title{
Baseline assessment of adult and adolescent primary care delivery in Rwanda: an opportunity for quality improvement
}

Ashwin Vasan ${ }^{1,2,3^{*}}$, Manzi Anatole ${ }^{1,4^{*}}$, Catherine Mezzacappa ${ }^{1,5}$, Bethany L Hedt-Gauthier ${ }^{1,4,5}$, Lisa R Hirschhorn ${ }^{1,5,6}$, Fulgence Nkikabahizi ${ }^{7}$, Marc Hagenimana ${ }^{7}$, Aphrodis Ndayisaba' ${ }^{1}$, Felix R Cyamatare ${ }^{1}$, Bonaventure Nzeyimana ${ }^{7}$, Peter Drobac ${ }^{1,5,6}$ and Neil Gupta ${ }^{1,5}$

\begin{abstract}
Background: As resource-limited health systems evolve to address complex diseases, attention must be returned to basic primary care delivery. Limited data exists detailing the quality of general adult and adolescent primary care delivered at front-line facilities in these regions. Here we describe the baseline quality of care for adults and adolescents in rural Rwanda.
\end{abstract}

Methods: Patients aged 13 and older presenting to eight rural health center outpatient departments in one district in southeastern Rwanda between February and March 2011 were included. Routine nurse-delivered care was observed by clinical mentors trained in the WHO Integrated Management of Adolescent \& Adult Illness (IMAI) protocol using standardized checklists, and compared to decisions made by the clinical mentor as the gold standard.

Results: Four hundred and seventy consultations were observed. Of these, only $1.5 \%$ were screened and triaged for emergency conditions. Fewer than $10 \%$ of patients were routinely screened for chronic conditions including HIV, tuberculosis, anemia or malnutrition. Nurses correctly diagnosed $50.1 \%$ of patient complaints (95\% Cl: $45.7 \%-54.5 \%$ ) and determined the correct treatment $44.9 \%$ of the time (95\% Cl: 40.6\%-49.3\%). Correct diagnosis and treatment varied significantly across health centers ( $p=0.03$ and $p=0.04$, respectively).

Conclusion: Fundamental gaps exist in adult and adolescent primary care delivery in Rwanda, including triage, screening, diagnosis, and treatment, with significant variability across conditions and facilities. Research and innovation toward improving and standardizing primary care delivery in sub-Saharan Africa is required. IMAI, supported by routine mentorship, is one potentially important approach to establishing the standards necessary for high-quality care.

Keywords: Primary care, Africa, Resource-limited settings, Quality improvement, Training, Integration, IMAI, Outpatient department, Nurses

\section{Background}

The growing success in resource-limited settings of disease-specific interventions for HIV, TB, malaria and other conditions has led to renewed focus on primary care. Integration of disease-specific services into primary care settings has emerged as a strategy to harness specific gains for more generalized improvement in

\footnotetext{
* Correspondence: ashwin@postharvard.edu; mangano2020@gmail.com 'Partners In Health-Inshuti Mu Buzima, Kigali, Rwanda and Boston, USA ${ }^{2}$ Department of Clinical Research, London School of Hygiene and Tropical Medicine, London, UK

Full list of author information is available at the end of the article
}

health care delivery, with HIV and reproductive health care most commonly studied [1-7]. Despite this work, relatively little attention has been paid directly to strengthening primary care services for adults and adolescents - the core services with which disease-specific programs should integrate. In many developing-world health systems, particularly in sub-Saharan Africa, the majority of general adult and adolescent ambulatory care occurs in the outpatient department (OPD), which typically operates within front-line health centers (HCs) or as auxiliary clinics to district or regional hospitals. In many settings, the OPD is the first point-of-contact with

\section{Biomed Central}


formalized health infrastructure for patients who are not already enrolled in other specialized ambulatory services (e.g. antenatal or TB care), or care for specific chronic diseases (e.g. HIV). As such, the OPD serves a critical function in the health system through triaging for life-threatening conditions, screening for chronic diseases and modifiable risk factors, diagnosis of acute illness, and appropriate management of both acute and chronic conditions. However, there are currently no benchmarks or targets established to define quality of care for adults and adolescents for most conditions in this primary care setting.

Few rigorous studies have been done on how to develop a standardized, integrated approach across conditions for the initial adult patient screening and work-up in resource-limited settings $[8,9]$. The few existing studies suggest that standardization and integration could be an important component of improving primary care and health worker performance [10-14], but more research is needed. Aside from disease-specific analyses and one qualitative survey [15] a literature review did not identify any reports describing the quality of general ambulatory primary care for adults and adolescents in any resourcelimited setting.

Many tools have been developed for services for selected conditions delivered within OPDs, including malaria, STIs, and TB. In addition, an integrated guideline for respiratory disease was developed and tested in southern Africa [16-18]. The World Health Organization (WHO) and partners have attempted to standardize general adult and adolescent primary care services, called IMAI - the Integrated Management of Adolescent \& Adult Illness [19]. Building off the success of the Integrated Management of Childhood Illness (IMCI) for children under-five years [20], IMAI is targeted for use by front-line health care workers, and consists of simplified, syndromic clinical protocols within a single integrated guideline for common presenting illnesses in adults and adolescents. Like IMCI, these syndromic protocols are organized around symptoms (e.g. fever, cough, diarrhea, etc.) and include a simplified approach to patient history and physical exam. These protocols then provide prescriptive guidance on the management (triage, screening, diagnose, treatment, and follow-up and referral) for a variety of conditions commonly seen in adult outpatient care. Unlike IMCI [21-26], IMAI has undergone little study, with limited, mostly unpublished validations from South Africa Simoes E, et al: Preliminary Analysis of IMAI Validation Studies. data.], Ethiopia [27], and Lesotho [Seung KJ, et al: Validation of Integrated Management of Adolescent and Adult Illness (IMAI) guidelines for patients with respiratory symptoms in Lesotho. Unpublished.].

The objective of this study is to describe the routine quality of care for adults and adolescents at OPDs in a rural district in Rwanda in order to document the current quality of care, identify needs for improvement, and propose possible interventions going forward.

\section{Methods \\ Study setting}

The study was conducted in southern Kayonza District, in Rwanda's Eastern Province, with an estimated total population of approximately 190,000 people [28]. The study was conducted at all eight of the Ministry of Health $(\mathrm{MOH})$ health centers that fall under the district hospital catchment area, and all of which receive support from Partners In Health-Inshuti Mu Buzima (PIH-IMB). PIH-IMB is a non-governmental organization partnering with the $\mathrm{MOH}$ since 2005 to strengthen health systems in three rural districts in Rwanda. Rwandan HCs are generally staffed by nurses holding the equivalent of a secondary school education in nursing. These nurses undergo no specific training in adult and adolescent primary care aside from their degree program and practice experience. There are, however, a number of disease-specific didactic trainings provided by the $\mathrm{MOH}$ and partners that some nurses may attend. Routine documentation of delivery of care is done in the OPD register, a standard lined registry that contains basic demographic, diagnostic and treatment data.

\section{Study population}

The study was conducted between February 1, 2011, and March 31, 2011. Consecutive patients 13 years or older presenting to the OPD were eligible for the study. There is no clearly defined age cut-off between childhood and adolescence in Rwanda [29]. Age 13 was chosen for this study based on definitions used by the United States' Centers for Disease Control (CDC) [30]. Data at each health center were collected over one week, with all centers visited in eight successive weeks.

\section{Data collection}

Data were collected by a clinical mentor, employed by the $\mathrm{MOH}$ and assigned to train and supervise OPD nurses as part of a larger initiative to improve quality of care at health centers in the district [31]. Supervision and clinical mentoring activities had not yet begun at the time of baseline data collection. The clinical mentor had the equivalent of a bachelor's degree in nursing with five years of relevant clinical experience. The clinical mentor underwent extensive didactic and practical training in the adapted IMAI protocols, mentoring and quality improvement. The standard WHO-IMAI protocols [32] were previously adapted and translated by the study staff and district $\mathrm{MOH}$ partners through consensus guideline review and pilot testing so as to adhere to Rwandan 
national treatment guidelines and reflect local epidemiologic priorities.

Data were collected by direct observation of routine clinical consultations using a standard data collection tool developed to document critical components of the clinical encounter. This observation checklist was structured upon the WHO-IMAI Case Management Observation Form, and included the standard triage checklist for emergency conditions contained within the IMAI Quick Check protocol, part of the larger IMAI guideline [19]. IMAI had not been formally introduced in Rwanda at the time of the study and study nurses had not received training in the adapted IMAI protocols.

The clinical mentor was present in the room during the consultation but was instructed to not intervene in patient care, with the exception of a critically ill patient requiring immediate action. Data collected in the observation checklist are summarized in Table 1. Formal diagnostic and management decisions made by the nurse were recorded by the clinical mentor from observed actions and review of the OPD register.

The clinical mentor recorded information on up to three chief complaints for each patient consultation. For each patient complaint the nurse, following $\mathrm{MOH}$ standards, selected from the $57 \mathrm{MOH}$ Système d'Information Sanitaire (SIS) diagnosis codes used in routine practice. The clinical mentor recorded the SIS diagnosis selected by the nurse and selected his own SIS diagnosis based on his clinical expertise and additional training in IMAI.

\section{Data analysis}

The primary outcome of interest was correct diagnosis of patient illness, defined as agreement in diagnosis between the nurse and the clinical mentor, with patient complaint as the unit of observation. For example, a patient complaining of both cough and back pain would receive one diagnosis for each complaint, and contribute two observations to the sample. Cases where the nurse found no appropriate diagnosis within the SIS codes for a given patient complaint were excluded from analysis of diagnosis agreement. Chief complaints were defined as the primary complaint either: (1) offered by the patient voluntarily, (2) given by the patient in response to direct questioning by the nurse, or (3) selected by the nurse independently at any point during the consultation.

Correct treatment of each complaint was also evaluated, defined as $100 \%$ agreement between the nurse and clinical mentor in all elements of treatment plan, including labs/diagnostic tests ordered, medications prescribed, referrals or transfers made, and follow-up recommendations. The nurse treatment plan reflected their routine practice, while the clinical mentor's treatment plan was based on the modified IMAI protocols. Observations where the nurse diagnosis was incorrect were not
Table 1 Summary of data categories within the study Observation Checklist

\begin{tabular}{|c|c|}
\hline 1. & Demographics \\
\hline \multirow[t]{8}{*}{2.} & Vital Signs \\
\hline & - Height \\
\hline & - Weight \\
\hline & - Pulse \\
\hline & - Respiratory Rate \\
\hline & - Temperature \\
\hline & - Blood pressure \\
\hline & - BMI (optional) \\
\hline \multirow[t]{2}{*}{3.} & Triage and Identification of Emergency Conditions \\
\hline & $\begin{array}{l}\text { - Based on IMAI Quick Check triage protocol within } \\
\text { WHO-IMAI guideline }\end{array}$ \\
\hline \multirow[t]{13}{*}{4.} & Screening \\
\hline & - Chronic cough \\
\hline & - Malnutrition \\
\hline & - Anemia \\
\hline & - Genital/anal lesions \\
\hline & $\begin{array}{l}\text { - Discharge/other genitourinary symptoms in males with genital } \\
\text { lesions }\end{array}$ \\
\hline & - Previous HIV testing \\
\hline & - Mosquito net \\
\hline & - Tobacco use \\
\hline & - Alcohol use \\
\hline & - Sexual activity \\
\hline & - Pregnancy in eligible females \\
\hline & - Family planning in females \\
\hline 5. & Chief Complaint(s) \\
\hline \multirow[t]{2}{*}{6.} & Diagnosis \\
\hline & - Based on MOH SIS diagnostic codes \\
\hline \multirow[t]{2}{*}{7.} & Treatment \\
\hline & $\begin{array}{l}\text { - } 100 \% \text { agreement, including labs, medications, and other } \\
\text { treatments ordered }\end{array}$ \\
\hline \multirow[t]{7}{*}{8.} & Referrals \\
\hline & - Including follow-up visits to the OPD, if any \\
\hline & - Patient Age and Sex \\
\hline & - Start and end time of consultation \\
\hline & - Health center \\
\hline & - Nurse education level \\
\hline & - Years of OPD experience of nurse \\
\hline
\end{tabular}

automatically excluded from the treatment analysis, so as to capture those cases where the nurse may have selected an incorrect diagnosis but still made a correct treatment plan.

Data were double-entered into a Microsoft Access database and analyzed using SASv.9.2 (Cary, NC, USA). 
The sample was described using frequencies for categorical variables and means for continuous variables. Patient assessment by nurses was described using frequencies of vital sign measurement, triage, and screenings. The primary outcomes - frequencies of correct nurse diagnosis and correct nurse treatment - were calculated for each complaint, along with corresponding $95 \%$ confidence intervals. A secondary analysis was conducted to identify factors associated with the quality of nurse case management. Relationships between correct diagnosis and treatment and health center and duration of nurse experience in OPD dichotomized at four years (the sample median) were assessed using Pearson's chisquared tests.

\section{Ethics}

This study was approved by the Rwandan National Ethics Committee and the Institutional Review Boards (IRB) at Partners Healthcare and the London School of Hygiene \& Tropical Medicine. Data were collected as part of routine program monitoring of an ongoing mentorship and quality improvement intervention in the study district. No identifying nurse or patient information was collected. As such, the IRB approved a waiver of informed consent under the routine use of program monitoring data. However, patients were explained the purpose of the data collection and could opt-out if desired.

\section{Results}

A total of 470 patient consultations were observed (range 53-69 per health center). The median duration of consultation was six minutes (Interquartile Range (IQR): 5-8 minutes) Approximately two-thirds (68.4\%) were female and mean patient age was 35.3 years (standard deviation: 15.9 years). All nurses performing the consultations had the nursing education described above. The median practice experience of the nurses was four years (IQR:3-6 years) (Table 2).

\section{Chief complaints}

The majority of patients (75.3\%) had one chief complaint, with $21.7 \%(\mathrm{n}=102)$ reporting two complaints, $1.9 \%(\mathrm{n}=9)$ reporting three complaints, and $1.1 \%(\mathrm{n}=5)$ with no complaint recorded. The most common chief complaints were female genitourinary issues (15.0\%), cough (14.2\%), epigastric abdominal pain (11.1\%), and fever $(9.7 \%)$. Of note, only $13.1 \%(n=61)$ of patients were asked directly for a chief complaint by the nurse (Table 2).

\section{Vital signs}

At least one of six vital signs was recorded by any staff at $70.9 \%$ of visits, with no visits having all seven vital signs
Table 2 Sample characteristics and distribution of patient complaints

\begin{tabular}{ll}
\hline Number of observed consultations & $\mathbf{4 7 0}$ \\
\hline Nurse years of experience in OPD, median (IQR) & $4(3-6)$ \\
Duration (minutes), median (IQR) & $6(5-8)$ \\
Patient sex, n (\%) & \\
$\quad$ Female & $319(68.4 \%)$ \\
$\quad$ Male & $147(31.6 \%)$ \\
Patient age (years), mean (s.d.) & $35.3(15.9)$ \\
Patients asked why they came to OPD, n (\%) & $61(13.1 \%)$ \\
Total number of patient complaints & 585 \\
Zero (0) complaints & $5(1.1 \%)$ \\
One (1) complaint & $354(75.3 \%)$ \\
Two (2) complaints & $102(21.7 \%)$ \\
Three (3) complaints & $9(1.9 \%)$ \\
Back or joint pain & $44(7.5 \%)$ \\
Cough or difficulty breathing & $83(14.2 \%)$ \\
Diarrhea & $31(5.3 \%)$ \\
Epigastric pain & $65(11.1 \%)$ \\
Fever & $58(9.9 \%)$ \\
Genital or anal sore, ulcer, or wart & $5(0.9 \%)$ \\
Genitourinary symptoms & \\
Female & $32(5.5 \%)$ \\
Meadache or neurological condition & $43(7.4 \%)$ \\
\hline Hypertension & $32(5.5 \%)$ \\
Lower extremity edema & $52(8.9 \%)$ \\
Mental problem & $3(0.5 \%)$ \\
\hline & $6(1.0 \%)$ \\
\hline & $6(1.0 \%)$ \\
Skoth or throat problem & $37(6.3 \%)$ \\
\hline
\end{tabular}

taken (Table 3). At least one vital sign was taken by a registration clerk prior to seeing the nurse in $56.0 \%$ of visits, and in $11.5 \%$ of patient visits, more than one provider collected vital signs. Weight was the most commonly taken vital sign (62.6\%). Temperature was recorded for $27.5 \%$ of patients, and $16.2 \%$ had blood pressure recorded. No patient had height, respiratory rate or pulse recorded, nor body-mass index (BMI) calculated. One HC had no available registration clerk during any of the patient care sessions observed.

\section{Triage}

Only seven (1.5\%) patients were fully screened and triaged based on the standard triage protocol within IMAI. Five patients (1\%) required higher level of care per the clinical mentor, of whom four were appropriately diagnosed and managed by the nurse (Table 3 ). 
Table 3 Quality Indicators - Consultations with Vital Signs, Triage and Screenings by Outpatient Department nurse $(\mathrm{N}=470)^{\mathrm{a}}$

\begin{tabular}{|c|c|}
\hline \multicolumn{2}{|l|}{ Vital signs collected by: } \\
\hline Registration clerk & $209(44.5 \%)$ \\
\hline Outpatient Department nurse & $118(25.1 \%)$ \\
\hline Other & $75(16.0 \%)$ \\
\hline Multiple providers & $54(11.5 \%)$ \\
\hline None & $14(3.0 \%)$ \\
\hline Blood pressure recorded & $76(16.2 \%)$ \\
\hline Temperature recorded & $129(27.5 \%)$ \\
\hline Weight recorded & $294(62.6 \%)$ \\
\hline Height recorded & 0 \\
\hline Respiratory rate recorded & 0 \\
\hline BMI recorded & 0 \\
\hline Pulse recorded & 0 \\
\hline \multicolumn{2}{|l|}{ Triage } \\
\hline $\begin{array}{l}\text { Triaged using basic skills in assessment of } \\
\text { severe illness }\end{array}$ & $7(1.5 \%)$ \\
\hline Diagnosed with emergency condition by nurse & $4(0.9 \%)$ \\
\hline \multicolumn{2}{|l|}{ Screening } \\
\hline Chronic cough & $45(10.0 \%)$ \\
\hline Malnutrition & $1(0.2 \%)$ \\
\hline Anemia & $8(1.8 \%)$ \\
\hline Genital/anal lesions & $17(3.8 \%)$ \\
\hline \multirow{2}{*}{$\begin{array}{l}\text { Discharge/other genitourinary symptoms in males } \\
\text { with genital lesions }(n=129)\end{array}$} & 12 (9.3\%) penile \\
\hline & $1(0.8 \%)$ scrotal \\
\hline Previous HIV testing & $7(1.8 \%)$ \\
\hline Mosquito net & $3(0.7 \%)$ \\
\hline Tobacco use & $2(0.4 \%)$ \\
\hline Alcohol use & $2(0.4 \%)$ \\
\hline Sexual activity & $32(7.3 \%)$ \\
\hline Pregnancy in eligible females $(n=300)$ & $66(22.0 \%)$ \\
\hline Family planning $(n=268)^{b}$ & $10(3.7 \%)$ \\
\hline
\end{tabular}

anless otherwise indicated.

bonly women aged 15-49 eligible for screening per adapted IMAI protocol.

All four patient emergencies identified by the nurse were referred to the district hospital; three with high fevers greater than $38^{\circ} \mathrm{C}$ and one who was convulsing. One patient, identified only by the clinical mentor, was in circulatory shock and with mentor intervention, resuscitation measures were employed.

\section{Routine screening by history}

Low rates were observed for routine screening for a wide range of conditions (Table 3). Only 10\% (45) of patients were screened for $\mathrm{TB}$ (cough greater than 2 weeks). Among male patients, $10.1 \%$ were screened for genitourinary/STI symptoms. Twenty-two percent
(66 of 300) of eligible female patients were screened for pregnancy or asked about their last menstrual period. Only 7.3\% (32) of patients were asked about current sexual activity, and only $1.8 \%$ (7) screened for HIV status. Less than $1 \%$ of patients were screened for any of the following: anemia, alcohol use, tobacco use, use of mosquito nets at home or nutrition.

\section{Diagnosis and treatment}

In the 470 consultations observed, patients reported a total of 585 complaints. Of these, forty-nine categorized as "Other" $(n=43)$ and "Mental problem" $(n=6)$ were not captured by the disease categories included in the checklist and were excluded from analysis. Seven additional complaints (1.2\%) had no corresponding diagnosis in the SIS codes, and 32 (5.5\%) were missing any recorded diagnosis information, leaving 497 complaints with a diagnosis available for analysis. In addition to the 49 excluded complaints described above, 33 (5.6\%) observations were missing information on correct treatment, leaving 503 complaints with available treatment data for comparison (Table 4).

Nurse diagnoses were in agreement with the clinical mentor $50.1 \%$ of the time (95\% confidence interval $45.7 \%-54.5 \%$ ), with the highest diagnosis agreement seen for epigastric pain (90.0\%), skin problems or lumps (83.9\%), mouth or throat problems $(70.3 \%)$, and diarrhea $(70.0 \%)$. Lowest rates of diagnosis agreement were seen for fever $(8.2 \%$ correct), headache or neurological

Table 4 Correct nurse diagnosis and treatment of patient illnesses*

\begin{tabular}{|c|c|c|c|}
\hline & & $\begin{array}{l}\text { Nurse diagnosis } \\
\quad \mathrm{n}=497\end{array}$ & $\begin{array}{l}\text { Nurse treatment } \\
n=503\end{array}$ \\
\hline & $\mathrm{N}$ & $\%(95 \% \mathrm{Cl})^{\ddagger}$ & $\%(95 \% \mathrm{Cl})$ \\
\hline \multirow[t]{2}{*}{ Total agreement } & & 249 & 226 \\
\hline & & $50.1 \%(45.7-54.5)$ & $44.9 \%(40.6-49.3)$ \\
\hline Back or joint pain & 42 & $59.5 \%(44.7-74.4)$ & $71.4 \%(57.8-85.1)$ \\
\hline $\begin{array}{l}\text { Cough or difficulty } \\
\text { breathing }\end{array}$ & 80 & $31.3 \%(21.1-41.4)$ & $33.8 \%(23.4-44.1)$ \\
\hline Diarrhea & 30 & $70.0 \%(53.6-86.4)$ & $36.7 \%(19.4-53.9)$ \\
\hline Epigastric pain & 59 & $90.0 \%(82.4-97.6)$ & $18.6 \%(8.7-28.6)$ \\
\hline Fever & 49 & $8.2 \%(2.3-19.6)$ & $78.6 \%(67.8-89.3)$ \\
\hline \multicolumn{4}{|l|}{ Genitourinary symptoms } \\
\hline Female & 80 & $48.8 \%(38.0-59.6)$ & $35.0 \%(24.6-45.5)$ \\
\hline Male & 30 & $53.3 \%(35.5-71.1)$ & $26.7 \%(10.8-42.5)$ \\
\hline $\begin{array}{l}\text { Headache or neurological } \\
\text { condition }\end{array}$ & 44 & $20.5 \%(8.5-32.4)$ & $77.3 \%(64.9-89.7)$ \\
\hline Mouth or throat problem & 37 & $70.3 \%(55.5-85.0)$ & $43.2 \%(27.3-59.2)$ \\
\hline Skin problem or lump & 31 & $83.9 \%(70.9-96.8)$ & $45.2 \%(27.6-62.7)$ \\
\hline
\end{tabular}

*Limited to complaints with $\mathrm{n}>5$ observations.

${ }^{\ddagger} 95 \% \mathrm{Cl}$ calculated using the exact binomial distribution. 
conditions (20.5\%) and cough or difficulty breathing (31.3\%). Agreement varied significantly between HCs, ranging from $35.6 \%$ to $60.0 \%(\mathrm{p}=0.03)$. There was no association between nurse experience in OPD and correct diagnosis.

The nurse treatment plan was in agreement with the clinical mentor less than half the time $(44.9 \%, 95 \%$ confidence interval $40.6 \%$ - 49.3\%). Complaints of fever (78.6\%), headache $(77.3 \%)$, and back or joint pain (71.4\%) had the highest proportion of agreement. Lowest agreement was seen for epigastric pain (18.6\%), genitourinary symptoms (26.7\% for males, $35.0 \%$ for females), cough or difficulty breathing (33.8\%), and diarrhea (36.7\%). Treatment agreement varied from $32.1 \%$ to $58.9 \%$ across HCs $(\mathrm{p}=0.04)$, but did not vary with duration of nurse experience in the OPD (Table 5).

\section{Discussion}

This is, to our knowledge, the first study assessing the general quality of care and clinical decision-making for adults and adolescents at rural OPDs in a resourcelimited setting. Every aspect of routine care that was measured, including triage for life-threatening conditions, screening for chronic disease and modifiable lifestyle risk-factors, diagnosis of acute illness, and appropriate management and treatment, were found to be in need of strengthening and quality improvement. The study demonstrated infrequent collection and use of vital signs, infrequent screening for common illnesses (such as HIV, TB, STIs), and low rates of prevention counseling (such as malaria control with ITNs, and alcohol and tobacco use). With a lack of available standards to support quality, compounded by relatively little investment in general primary care for adults and

Table 5 Factors associated with correct nurse diagnosis and treatment of patient illness

\begin{tabular}{|c|c|c|c|c|c|c|}
\hline & \multicolumn{3}{|c|}{ Nurse diagnosis } & \multicolumn{3}{|c|}{ Nurse treatment } \\
\hline & $\mathrm{N}$ & Percent correct & $p$ & $\bar{N}$ & Percent correct & $p$ \\
\hline Health center & & & 0.03 & & & 0.04 \\
\hline$A$ & 54 & $51.9 \%(38.5-65.2)$ & & 56 & $58.9 \%(46.0-71.8)$ & \\
\hline$B$ & 61 & $37.7 \%(25.5-49.9)$ & & 61 & $42.6 \%(30.2-55.0)$ & \\
\hline C & 62 & $58.1 \%(45.8-70.4)$ & & 64 & $43.8 \%(31.6-55.9)$ & \\
\hline D & 65 & $60.0 \%(48.1-71.9)$ & & 65 & $32.3 \%(20.9-43.7)$ & \\
\hline$E$ & 68 & $58.8 \%(47.1-70.5)$ & & 69 & $50.7 \%(38.9-62.5)$ & \\
\hline $\mathrm{F}$ & 59 & $35.6 \%(23.4-47.8)$ & & 59 & $52.5 \%(39.8-65.3)$ & \\
\hline G & 55 & $49.1 \%(35.9-62.3)$ & & 56 & $32.1 \%(19.9-44.4)$ & \\
\hline$H$ & 73 & $46.6 \%(35.1-58.0)$ & & 73 & $46.6 \%(35.1-58.0)$ & \\
\hline $\begin{array}{l}\text { Nurse OPD } \\
\text { experience }\end{array}$ & & & 0.26 & & & 0.66 \\
\hline $4+$ years & 264 & $51.5 \%(45.5-57.5)$ & & 265 & $43.8 \%(37.8-49.8)$ & \\
\hline $0-3$ years & 220 & $46.4 \%(39.8-53.0)$ & & 225 & $45.8 \%(39.3-52.3)$ & \\
\hline
\end{tabular}

adolescents, it is unsurprising that the results reflect a great need for improvement of front-line delivery.

Nurse diagnosis and treatment decisions were in agreement with the clinical mentor approximately half of the time or less. Agreement in diagnosis and treatment for the same conditions (e.g. fever, epigastric pain) often varied widely, suggesting large variations in individual practice. Poor decision-making could be influenced by a number of long-term structural and health system needs, including gaps in infrastructure, supplies and human resources [33-36]. However, other factors could be addressed in the shorter-term and could lead to an initial rapid improvement in the quality of adult and adolescent primary care.

As noted, aside from selected disease- or conditionspecific protocols there are few decision-support tools available to help OPD providers simplify and standardize their decision-making and care. These tools, particularly for children under-5 and for HIV/AIDS, have been shown to improve quality and health worker performance [37-42]. While experiential learning is the primary foundation of practice improvement in Rwandan OPDs, the results show there was no significant increase in the quality of the decision making with increasing years of nursing experience.

The data also highlight needs with respect to nurse training and standardization of care. The low frequencies in our data across the measured areas of clinical practice suggest that the nurses in our sample need additional support in basic clinical skills, including history-taking, completion and interpretation of vital signs, and screening for chronic conditions, in addition to clinical reasoning and decision-making. While it was beyond the scope of this study to isolate whether these gaps originated in preservice education, pre- or post-service training, and/or other structural factors such as infrastructure, lack of protocols or time constraints, clinical training of nurses in primary care has been shown to at least modestly improve quality of care for acute respiratory tract infections [43] and medication prescription [44]. The data demonstrate that nurses manage some illnesses better than others, and that performance differs significantly between sites, suggesting that standardization of care, as well as performance measurement coupled with mentoring and systems-based quality improvement are needed. In addressing issues of decision support, integrated training, and standardization of care, protocols such as IMAI could represent an important part of a toolkit for developing a common standard of OPD care for adults and adolescents, and improving quality of these services.

While implementation of care based on protocols such as IMAI is necessary, it is not sufficient to support the delivery of high quality of care. A critical component is ensuring durable knowledge and practice improvement 
through routine and sustainable mentorship of health care workers. Though there are differences in definition and structure of supervision programs [45], mentorship in primary care has been shown to improve and sustain improvements in nurse performance [46-48]. In Rwanda, robust efforts are now underway to implement a system of routine on-site mentorship to health center nurses in all clinical services [31].

There are a number of limitations to our study. First the clinical mentor was not blinded and therefore could have been influenced by observing nurses' decisions in real-time, thus biasing the results and possibly overestimating the level of agreement. Having an observer in the room may have also influenced the nurses to perform better or differently than they would in normal circumstances [49]. Given the low level of consistency found, it is unlikely that either potential bias considerably altered the results. Also, clinical decision-making could not be assessed for approximately $15 \%$ of patient complaints due to missing data. Approximately half $(n=43)$ of complaints missing from the analysis were classified as "Other" complaints that did not fall into the conditions selected for study a priori. The remainder of missing diagnosis or treatment information (approximately $7 \%$ of the total sample) was distributed across complaints and is unlikely to have had meaningful impact on the results. We have also presented clinical decision-making on diagnosis and therapeutics as a proxy for quality of care, but these are only two key components. We did not measure clinical outcomes, nor define current costs, infrastructure, and human resources which all impact or help define quality and which could be pursued in future research. Finally, IMAI has yet to be formally validated, particularly in low-HIV prevalence settings such as Rwanda, which represents an important next step in establishing a standard of general adult and adolescent primary care delivery.

\section{Conclusions}

This study describes the baseline quality of adult and adolescent primary care at Rwandan health center OPDs and demonstrates a clear need for improving the quality and consistency of care. Care based on IMAI or other integrated protocols, accompanied by a robust system of mentorship and supervision that focuses on individual performance as well as systems-level gaps, could be an important approach to simplifying and standardizing the delivery of care for common acute presentations for adults and adolescents in the ambulatory setting. Refining and validating these protocols is needed. Only these coordinated efforts can ensure that high quality and effective primary care is available for all adults and adolescents in resource limited settings.

\section{Competing interests}

All listed authors declare that there are no competing interests - financial or otherwise - with respect to the design or execution of this study, or the writing or publication of the manuscript.

\section{Authors' contributions}

$A V$ led the principle design of the study including development of data collection tools, data analysis plan, and served as overall co-lead supervisor for the study with MA, and was responsible for writing the first and all subsequent drafts of the manuscript in tandem with MA as well as incorporating authors' editorial comments. MA was the co-lead supervisor for the study working directly with AV on the study design, and served as the principle field coordinator and field supervisor for the study, refining of data collection tools and techniques, and was responsible with AV for drafting the first and all subsequent drafts of the manuscript, especially the Results and Discussion. CM served as the principal data analyst for the study, developing the study database and leading data entry, and conducted the main data analysis for the study according to the analysis plan, and as well contributing significant inputs to early and all subsequent versions of the manuscript, especially the Methods and Results. BLH was the principle statistician for the study, supporting data collection and analysis, and making significant written and editorial inputs to later versions of the manuscript, especially the Methods, Results, and Discussion. LRH is the Co-Principal Investigator for the larger DDCF research grant, and on this study served both as the senior technical advisor to the lead authors of the study, but also provided significant editorial inputs to all sections of later versions of the manuscript. FN served as the lead district $\mathrm{MOH}$ representative on the study and was involved in review and approval of the study protocol and all related study tools and procedures, and played an instrumental role in educating staff at participating sites on the study, while also contributing significant editorial inputs and approval on later drafts of the manuscript. MH was the senior nurse clinical mentor and principal data collector on the study, was involved in development and field-testing of all data tools, and contributed significant editorial inputs to later drafts of the manuscript, especially the Methods section. AN served as the deputy field coordinator for the study under MA and was the principle point of contact for all study staff on the ground and managed all of the study logistics, especially for the data collection and study implementation teams; he also contributed significant editorial inputs and approval for later versions of the manuscript. FRC served as the principle clinical lead on the study, working directly with the nurse mentor during the field testing and data collection stages, and also worked closely with the lead authors on design and field testing of the data collection tools, while also contributing significant editorial inputs and approval for later drafts of the manuscript. BN was the central $\mathrm{MOH}$ representative for the study, was instrumental in securing national ethics approval in Rwanda, and was involved particularly at the early stages of the study during design, approval and establishment of district partnership for the study, while also contributing significant editorial inputs to later drafts of the manuscript. PD is also a Co-PI on the larger DDCF grant, and served as the principle organizational lead on the study, involved particularly in the conception and design phases working directly with the lead authors, and provided significant editorial inputs and approval to later drafts of the manuscript, especially the Background, Results, and Discussion. NG served as the senior advisor on the study, involved at all stages of the study working in close collaboration with the lead authors, from design and conception to implementation and data management, and was instrumental in providing editorial inputs and approval to all drafts of the manuscript including the first draft. All authors read and approved the final manuscript.

\section{Authors' information}

Ashwin Vasan and Manzi Anatole are joint-first authors to this manuscript.

\section{Acknowledgments}

The study was conducted as part of the Rwanda Population Health Implementation \& Training (PHIT) Partnership, and funded by the Doris Duke Charitable Foundation's African Health Initiative. The Rwanda PHIT Partnership is co-led by the Rwanda Ministry of Health and Partners In Health with key involvement from the National University of Rwanda School of Public Health (NURSPH), Rwanda National Institute of Statistics, as well as Harvard Medical School, and Brigham and Women's Hospital in Boston, USA. $\mathrm{BHG}$ received additional support from the Department of Global Health and Social Medicine Research Core at Harvard Medical School. The authors would 
also like to thank Stephen D. Lawn and David C. Mabey from the London School of Hygiene \& Tropical Medicine for their helpful insight and comments.

\section{Author details}

'Partners In Health-Inshuti Mu Buzima, Kigali, Rwanda and Boston, USA. ${ }^{2}$ Department of Clinical Research, London School of Hygiene and Tropical Medicine, London, UK. ${ }^{3}$ Department of Medicine, Weill Cornell Medical College/New York-Presbyterian Hospital, New York, USA. ${ }^{4}$ University of Rwanda, College of Medicine and Health Sciences, School of Public Health, Kigali, Rwanda. ${ }^{5}$ Division of Global Health Equity, Brigham and Women's Hospital, Boston, USA. ${ }^{6}$ Department of Global Health and Social Medicine, Harvard Medical School, Boston, USA. ${ }^{7}$ Ministry of Health, Government of Rwanda, Kigali, Rwanda.

Received: 7 August 2013 Accepted: 4 December 2013 Published: 17 December 2013

\section{References}

1. World Health Organization Maximizing Positive Synergies Collaborative Group, Samb B, Evans T, Dybul M, et al: An assessment of interactions between global health initiatives and country health systems. Lancet 2009, 373(9681):2137-2169.

2. Rabkin M, El-Sadr WM: Why reinvent the wheel? Leveraging the lessons of HIV scale-up to confront non-communicable diseases. Glob Public Health 2011, 6:247-256

3. Mukherjee J, Eustache F: Community health workers as a cornerstone for integrating HIV and primary healthcare. AIDS Care 2007, 19(Suppl. 1):S73-S82.

4. Mayhew SH, Lush L, Cleland J, Walt G: Implementing the integration of component services for reproductive health. Stud Fam Plann 2000, 31:151-162.

5. Price JE, Leslie JA, Welsh M, Binagwaho A: Integrating HIV clinical services into primary health care in Rwanda: a measure of quantitative effects. AIDS Care 2009, 21(5):608-614.

6. Topp SM, Chipukuma JM, Chiko MM, et al: Integrating HIV treatment with primary care outpatient services: opportunities and challenges from a scaled-up model in Zambia. Health Policy Plan 2012, 28(4):347-357.

7. Topp SM, Chipukuma JM, Giganti M, et al: Strengthening health systems at facility-level: feasibility of integrating antiretroviral therapy into primary health care services in Lusaka, Zambia. PLoS One 2010, 5:e11522.

8. Dudley L, Garner P: Strategies for integrating primary health services in middle- and low-income countries at the point of delivery. Cochrane Database Syst Rev 2011, 7:CD003318.

9. Briggs CJ, Capdegelle P, Garner P: Strategies for integrating primary health services in middle- and low-income countries: effects on performance, costs and patient outcomes. Cochrane Database Syst Rev 2001, 4:CD003318.

10. Wallace A, Dietz V, Cairns KL: Integration of immunization services with other health interventions in the developing world: what works and why? Systematic literature review. Trop Med Int Health 2009, 14(1):11-9.

11. Seung K, Rigodon J, Finch M, Gove S, Vasan A, Satti H: Distribution of adult respiratory illnesses at a primary health centre in Lesotho. Int J Tuberc Lung Dis 2012, 16(3):418-22.

12. Frenk J: Reinventing primary health care: the need for systems integration. Lancet 2009, 374:170-3

13. Pinto RM, Wall M, Yu G, et al: Primary Care and Public Health Services Integration in Brazil's Unified Health System. Am J Public Health 2012, 102(11):e69-76.

14. Kruk ME, Porignon D, Rockers PC, et al: The contribution of primary care to health and health systems in low- and middle-income countries: a critical review of major primary care initiatives. Soc Sci Med 2010, 70(6):904-11.

15. Makaula P, Bloch P, Banda HT, et al: Primary health care in rural Malawi - a qualitativeassessment exploring the relevance of the communitydirected interventions approach. BMC Health Serv Res 2012, 12:328.

16. Bheekie A, Buskens I, Allen S, et al: The Practical Approach to Lung Health in South Africa (PALSA) intervention: respiratory guideline implementation for nurse trainers. Int Nurs Rev 2006, 53(4):261-8.

17. English RG, Bachmann MO, Bateman ED, et al: Diagnostic accuracy of an integrated respiratory guideline in identifying patients with respiratory symptoms requiring screening for pulmonary tuberculosis: a crosssectional study. BMC Pulm Med 2006, 6:22.
18. Sodhi S, Banda H, Kathyola D, et al: Evaluating a streamlined clinical tool and educational outreach intervention for health care workers in Malawi: the PALM PLUS case study. BMC Int Health Hum Rights 2011, 11(Suppl 2):S11.

19. World Health Organization: IMAI/IMCI heath centre/primary care guideline modules. Available at URL: http://www.who.int/hiv/pub/imai/primary/en/, (accessed June 10, 2013).

20. Gove S, for the WHO Working Group on Guidelines for Integrated Management of the Sick Child: Integrated management of childhood illness by outpatient health workers: technical basis and overview. Bull World Health Organ 1997, 75(suppl 1):7-24.

21. Weber MW, Mulholland ED, Jaffar S, et al: Evaluation of an algorithm for the integrated management of childhood illness in an area with seasonal malaria in the Gambia. Bull World Health Organ 1997, 75(suppl 1):25-32.

22. Perkins BA, Zucker JR, Otieno J, et al: Evaluation of an algorithm for integrated management of childhood illness in an area of Kenya with high malaria transmission. Bull World Health Organ 1997, 75(suppl1):33-42.

23. Kolstad PR, Burnham G, Kalter HD, et al: The integrated management of childhood illness in western Uganda. Bull World Health Organ 1997, 75(suppl 1):77-85.

24. Kalter HD, Burnham G, Kolstad PR, et al: Evaluation of clinical signs to diagnose anemia in Uganda and Bangladesh, in areas with and without malaria. Bull World Health Organ 1997, 75(suppl 1):103-11.

25. Simoes EA, Desta T, Tessema T, et al: Performance of health worker after training in integrated management of childhood illness in Gondar, Ethiopia. Bull World Health Organ 1997, 75(suppl 1):43-53.

26. Kahigwa E, Schellenberg D, Schellenberg JA, et al: Inter-observer agreement in the assessment of clinical signs in sick Tanzanian children. Trans R Soc Trop Med Hyg 2002, 96(2):162-6.

27. Woldie M, Enquselassie F: Assessment of the validity of the guideline for integrated management of adult and adolescent illnesses on HIV patients in Addis Ababa, Ethiopia. Ethiop Med J 2009, 47(3):195-203.

28. Government of Rwanda, National Institute of Statistics of Rwanda: 2012 Population and Housing Census (Provisional Results). Available from URL: http://www.statistics.gov.rw/publications/2012-population-andhousing-census-provisional-results. Accessed 9 May 2013.

29. Binagwaho A, Fuller A, Kerry V, Dougherty S, Agbonyitor M, Wagner C, Nzayizera R, Farmer P: Adolescents and the right to health: eliminating age-related barriers to HIV/AIDS services in Rwanda. AIDS Care 2012, 24(7):936-42.

30. CDC: Revised surveillance case definitions for HIV infection among adults, adolescents, and children aged $<18$ months and for HIV infection and AIDS among children aged 18 months to $<13$ years -- United States, 2008. MMWR 2008, 57(RR10):1-8.

31. Anatole $M$, Magge $H$, Redditt $V$, et al: Nurse mentorship to improve the quality of health care delivery in rural Rwanda. Nurs Outlook 2013, 61(3):137-44.

32. World Health Organization: IMAI Acute Care guideline. Available at URL: http://www.who.int/hiv/pub/imai/primary_acute/en/index.html.

33. World Health Organization: Working Together for Health: The World Health Report 2006. Geneva, Switzerland: World Health Organization; 2006.

34. Chen L, Evans T, Anand S, et al: Human resources for health: overcoming the crisis. Lancet 2004, 364:1984-1990.

35. Dussault G, Dubois CA: Human resources for health policies: a critical component in health policies. Hum Resour Health 2003, 1:1.

36. Wyss K: An approach to classifying human resources constraints to attaining health-related Millennium Development Goals. Hum Resour Health 2004, 2:11.

37. Amaral J, Gouws E, Bryce J, et al: Effect of Integrated Management of Childhood Illness (IMCl) on health worker performance in NortheastBrazil. Cad Saude Publica 2004, 20(Suppl 2):S209-19. Epub 2004 Dec 15.

38. El Arifeen S, Blum LS, Hoque DM, et al: Integrated Management of Childhood IIIness (IMCI) in Bangladesh: early findings from a clusterrandomised study. Lancet 2004, 364(9445):1595-1602.

39. Bryce J, Gouws E, Adam T, et al: Improving quality and efficiency of facility-based child health care through Integrated Management of Childhood Illness in Tanzania. Health Policy Plan 2005, 20(Suppl 1):i69-i76.

40. Mitchell M, Hedt BL, Eshun-Wilson I, et al: Electronic decision protocols for ART patient triaging to expand access to HIV treatment in South Africa: a cross sectional study for development and validation. Int J Med Inform 2012, 81(3):166-72. 
41. Edward A, Dwivedi V, Mustafa $L$, et al: Trends in the quality of health care for children aged less than 5 years in Afghanistan, 2004-2006. Bull World Health Organ 2009, 87(12):940-9.

42. Zhang Y, Dai Y, Zhang S: Impact of implementation of Integrated Management of Childhood Illness on improvement of health system in China. J Paediatr Child Health 2007, 43(10):681-5.

43. Louwagie GM, Bachmann MO, Reid M: Formal clinical primary health care training. Does it make a difference? Curationis 2002, 25(4):32-7.

44. Pagaiya N, Garner P: Primary care nurses using guidelines in Thailand: a randomized controlled trial. Trop Med Int Health 2005, 10(5):471-7.

45. Bosch-Capblanch X, Garner P: Primary health care supervision in developing countries. Trop Med Int Health 2008, 13:369-83.

46. Rowe AK, Onikpo F, Lama M, et al: A multifaceted intervention to improve health worker adherence to integrated management of childhood illness quidelines in Benin. Am J Public Health 2009, 99(5):837-46.

47. Horwood C, Vermaak K, Rollins N, et al: An evaluation of the quality of $\mathrm{IMCl}$ assessments among $\mathrm{IMCl}$ trained health workers in South Africa. PLoS One 2009, 17:4(6).

48. Rowe AK, Onikpo F, Lama M, Deming MS: The rise and fall of supervision in a project designed to strengthen supervision of Integrated Management of Childhood Illness in Benin. Health Policy Plan 2010, 25(2):125-34.

49. Leonard KL, Masatu MC: Outpatient process quality evaluation and the Hawthorne effect. Soc Sci Med 2006, 69(9):2330-2340.

doi:10.1186/1472-6963-13-518

Cite this article as: Vasan et al:: Baseline assessment of adult and adolescent primary care delivery in Rwanda: an opportunity for quality improvement. BMC Health Services Research 2013 13:518.

\section{Submit your next manuscript to BioMed Central and take full advantage of:}

- Convenient online submission

- Thorough peer review

- No space constraints or color figure charges

- Immediate publication on acceptance

- Inclusion in PubMed, CAS, Scopus and Google Scholar

- Research which is freely available for redistribution 